\title{
Analysis for prevalence of carpal tunnel syndrome in shocker manufacturing workers
}

\author{
Kumar, S. , Muralidhar, M. ${ }^{\mathrm{a},{ }^{*}}$ \\ ${ }^{a}$ Department of Mechanical Engineering, North Eastern Regional Institute of Science and Technology, Itanagar, Arunachal \\ Pradesh, India
}

\begin{abstract}
A B S T R A C T
Carpal tunnel syndrome (CTS) is the most commonly reported work-related musculoskeletal disorder of the upper extremity. In this communication, a comparison of CTS and associated risk factors amongst traditional and semiergonomic shocker manufacturing assembly line workers in the actual industrial environment has been studied through questionnaire and physical tests. Fisher's exact test and Surface electromyography (sEMG) signal values have been used for statistical data analysis. Symptoms present are numbness (in $80 \%$ of traditional and in $16.66 \%$ of semi-ergonomic), tingling (in $50 \%$ of traditional and in $8.33 \%$ of semi-ergonomic), and difficulty in grasping (in $80 \%$ of traditional and $20 \%$ of semi-ergonomic). Tinel's and Phalen's sign also show an almost similar trend. The results reflect that the traditional shocker manufacturing workers have more CTS symptoms occurrence than the semi-ergonomic shocker manufacturing workers. The sEMG signal analysis result reveals that the lesser muscle activity values (EMG-RMS values) indicate the contribution of CTS symptom in shocker assembly line workers. It is found that there is a significant difference in EMG-RMS values of CTS symptoms and control subjects in traditional and semi-ergonomic shocker manufacturing industries. It is observed that if a subject is affected with CTS symptoms, then the sEMG signal value is $0.01223 \mathrm{mV}$ in case of traditional and $0.02625 \mathrm{mV}$ in case of semi-ergonomic shocker assembly, and for control subjects SEMG signal value is $0.15614 \mathrm{mV}$ in traditional and $0.17563 \mathrm{mV}$ in case of semi-ergonomic shocker assembly.
\end{abstract}

\section{ARTICLE INFO}

Keywords:

Manufacturing workers

Musculoskeletal disorders

Carpal tunnel syndrome

Fisher's exact test

Surface electromyography

*Corresponding author:

mm@nerist.ac.in

(Muralidhar, M.)

Article history:

Received 3 August 2015

Revised 24 January 2016

Accepted 8 March 2016

\section{References}

[1] Sprigg, C.A., Stride, C.B., Wall, T.D., Holman, D.J., Smith, P.R. (2007). Work characteristics, musculoskeletal disorders, and the mediating role of psychological strain: A study of call center employees, Journal of Applied Psychology, Vol. 92, No. 5, 1456-1466, doi: 10.1037/0021-9010.92.5.1456.

[2] Schultz, G., Mostert, K., Rothmann, I. (2012). Repetitive strain injury among South African employees: The relationship with burnout and work engagement, International Journal of Industrial Ergonomics, Vol. 42, No. 5, 449-456, doi: 10.1016/j.ergon.2012.06.003.

[3] Robertson, V., Stewart, T. (2004). Risk perception in relation to musculoskeletal disorders (Research report), Health \& Safety Executive, London, UK.

[4] Harcombe, H., McBride, D., Derrett, S., Gray, A. (2009). Prevalence and impact of musculoskeletal disorders in New Zealand nurses, postal workers and office workers, Australian and New Zealand Journal of Public Health, Vol. 33, No. 5, 437-441, doi: 10.1111/j.1753-6405.2009.00425.x.

[5] Health and Safety Executive (2009). Self-reported work-related illness and workplace injuries in 2008/2009: Results from the labour force survey, from http://www.hse.gov.uk/statistics/overall/hssh0809.pdf accessed June 19, 2010. 
[6] Dunning, K.K., Davis, K.G., Cook, C., Kotowski, S.E., Hamrick, C., Jewell, G., Lockey, J. (2010). Costs by industry and diagnosis among musculoskeletal claims in a state workers compensation system: 1999-2004, American Journal of Industrial Medicine, Vol. 53, No. 3, 276-284, doi: 10.1002/ajim.20774.

[7] Silverstein, B.A., Hughes, R.E. (1996). Upper extremity musculoskeletal disorders at a pulp and paper mill, Applied Ergonomics, Vol. 27, No. 3, 189-194, doi: 10.1016/0003-6870(95)00076-3.

[8] Gorsche, R.G., Wiley, J.P., Renger, R.F., Brant, R.F., Gemer, T.Y., Sasyniuk, T.M. (1999). Prevalence and incidence of carpal tunnel syndrome in a meat packing plant, Occupational \& Environmental Medicine, Vol. 56, No. 6, 417-422, doi:10.1136/oem.56.6.417.

[9] Fagarasanu, M., Kumar, S. (2003). Carpal tunnel syndrome due to keyboarding and mouse tasks: a review, International Journal of Industrial Ergonomics, Vol. 31, No. 2, 119-136, doi: 10.1016/S0169-8141(02)00180-4.

[10] Babski-Reeves, K.L., Crumtpon-Young, L.L. (2002). Comparisons of measures for quantifying repetition in predicting carpal tunnel syndrome, International Journal of Industrial Ergonomics, Vol. 30, No. 1, 1-6, doi: 10.1016/S0169-8141(02)00072-0.

[11] Carnahan, B.J., Norman, B.A., Redfern, M.S. (2001). Incorporating physical demand criteria into assembly line balancing, IIE Transactions, Vol. 33, No. 10, 875-887, doi: 10.1080/07408170108936880.

[12] Xu, Z., Ko, J., Cochran, D.J., Jung, M.-C. (2012). Design of assembly lines with the concurrent consideration of productivity and upper extremity musculoskeletal disorders using linear models, Computers \& Industrial Engineering, Vol. 62, No. 2, 431-441, doi: 10.1016/j.cie.2011.10.008.

[13] Carayon, P., Smith, M.J., Haims, M.C. (1999). Work organization, job stress, and work-related musculoskeletal disorders, Human Factors, Vol. 41, No. 4, 644-663, doi: 10.1518/001872099779656743.

[14] Kumar, S., Muralidhar, M. (2016). Ergonomical study of hand-arm vibrational exposure in a gear manufacturing plant in India, In: 18 $18^{\text {th }}$ International Conference on Applied Human Factors and Ergonomics, Kuala Lumpur, Malaysia, 1-4.

[15] Ulin, S.S., Keyserling, W.M. (2004). Case studies of ergonomic interventions in automotive parts distribution operations, Journal of Occupational Rehabilitation, Vol. 14, No. 4, 307-326, doi: 10.1023/B:J00R.0000047432. $\underline{07837.64 .}$.

[16] Ferguson, S.A., Marras, W.S., Allread, W.G., Knapik, G.G., Vandlen, K.A., Splittstoesser, R.E., Yang, G. (2011). Musculoskeletal disorder risk as a function of vehicle rotation angle during assembly tasks, Applied Ergonomics, Vol. 42, No. 5, 699-709, doi: 10.1016/j.apergo.2010.11.004.

[17] Silverstein, B.A., Stetson, D.S., Keyserling, W.M., Fine, L.J. (1997). Work-related musculoskeletal disorders: Comparison of data sources for surveillance, American Journal of Industrial Medicine, Vol. 31, No. 5, 600-608, doi: 10.1002/(SICI)1097-0274(199705)31:5<600::AID-AJIM15>3.0.C0;2-2.

[18] Punnett, L., Gold, J., Katz, J.N., Gore, R., Wegman, D.H. (2004). Ergonomic stressors and upper extremity musculoskeletal disorders in automobile manufacturing: a one year follow up study, Occupational \& Environmental Medicine, Vol. 61, No. 8, 668-674, doi: 10.1136/oem.2003.008979.

[19] Keyserling, W.M., Sudarsan, S.P., Martin, B.J., Haig, A.J., Armstrong, T.J. (2005). Effects of low back disability status on lower back discomfort during sustained and cyclical trunk flexion, Ergonomics, Vol. 48, No. 3, 219-233, doi: $10.1080 / 0014013042000327689$.

[20] Bernard, B.P. (1997). Musculoskeletal disorders and workplace factors: A critical review of epidemiologic evidence for work-related musculoskeletal disorders of the neck, upper extremity, and low back, US Department of Health and Human Services, DHHS (NIOSH) Publication No. 97B141, Cincinnati, Ohio, USA.

[21] National Research Council and Institute of Medicine (2001). Musculoskeletal disorders and the workplace: Low back and upper extremities, The National Academies Press, Washington D.C., USA, doi: 10.17226/10032.

[22] Punnett, L., Wegman, D.H. (2004). Work-related musculoskeletal disorders: The epidemiologic evidence and the debate, Journal of Electromyography and Kinesiology, Vol. 14, No. 1, 13-23, doi: 10.1016/i.jelekin.2003.09.015.

[23] Vujica Herzog, N., Vujica Beharic, R., Beharic, A., Buchmeister, B. (2014). Ergonomic analysis of ophthalmic nurse workplace using 3D simulation, International Journal of Simulation Modelling, Vol. 13, No. 4, 409-418, doi: 10.25 07/IJSIMM13(4)2.265.

[24] Bland, J.D.P. (2007). Carpal tunnel syndrome, BMJ, Vol. 335, 343-346, doi: 10.1136/bmj.39282.623553.AD.

[25] Visser, L.H., Ngo, Q., Groeneweg, S.J.M., Brekelmans, G. (2012). Long term effect of local corticosteroid injection for carpal tunnel syndrome: A relation with electrodiagnostic severity, Clinical Neurophysiology, Vol. 123, No. 4, 838-841, doi: 10.1016/j.clinph.2011.08.022.

[26] Hlebs, S., Majhenic, K., Vidmar, G. (2014). Body mass index and anthropometric characteristics of the hand as risk factors for carpal tunnel syndrome, Collegium Antropologicum, Vol. 38, No. 1, 219-226.

[27] Kate, M. (1995). A nonsurgical approach to carpal tunnel syndrome, In: Proceedings of the International Forum on New Science, Fort Collins, Colorado, USA, 13-17.

[28] Jagga, V., Lehri, A., Verma, S.K. (2011). Occupation and its association with carpal tunnel syndrome - A review, Journal of Exercise Science and Physiotherapy, Vol. 7, No. 2, 68-78.

[29] Kulick, M.I., Gordillo, G., Javidi, T., Kilgore, E.S. Jr., Newmeyer III, W.L. (1986). Long-term analysis of patients having surgical treatment for carpal tunnel syndrome, The Journal of Hand Surgery, Vol. 11, No. 1, 59-66, doi: 10.1016/S0363-5023(86)80104-6.

[30] MacDermid, J.C., Wessel, J. (2004). Clinical diagnosis of carpal tunnel syndrome: a systematic review, Journal of Hand Therapy, Vol. 17, No. 2, 309-319, doi: 10.1197/j.jht.2004.02.015.

[31] Barandun, M., von Tscharner, V., Meuli-Simmen, C., Bowen, V., Valderrabano, V. (2009). Frequency and conduction velocity analysis of the abductor pollicis brevis muscle during early fatigue, Journal of Electromyography and Kinesiology, Vol. 19, No. 1, 65-74, doi: 10.1016/j.jelekin.2007.07.003.

[32] Bland, J.D.P. (2000). A neurophysiological grading scale for carpal tunnel syndrome, Muscle \& Nerve, Vol. 23, No. 8, 1280-1283, doi: 10.1002/1097-4598(200008)23:8<1280::AID-MUS20>3.0.CO;2-Y. 
[33] Liu, F., Carlson, L., Watson, H.K. (2000). Quantitative abductor pollicis brevis strength testing: reliability and normative values, Journal of Hand Surgery, Vol. 25, No. 4, 752-759, doi: 10.1053/ihsu.2000.6462.

[34] Nobuta, S., Sato, K., Komatsu, T., Miyasaka, Y., Hatori, M. (2005). Clinical results in severe carpal tunnel syndrome and motor nerve conduction studies, Journal of Orthopaedic Science, Vol. 10, No. 1, 22-26, doi: 10.1007/s00776004-0852-x.

[35] Olmo, G., Laterza, F., Presti, L.L. (2000). Matched wavelet approach in stretching analysis of electrically evoked surface EMG signal, Signal Processing, Vol. 80, No. 4, 671-684, doi: 10.1016/S0165-1684(99)00160-7.

[36] Reaz, M.B.I., Hussain, M.S., Mohd-Yasin, F. (2006). Techniques of EMG signal analysis: Detection, processing, classification and applications, Biological Procedures Online, Vol. 8, No. 1, 11-35, doi: 10.1251/bpo115.

[37] Imteyaz, A., Ansari, F., Dey, U.K. (2012). A review of EMG recording technique, International Journal of Engineering Science and Technology, Vol. 4, No. 2, 530-539.

[38] Day, S. (2002). Important factors in surface EMG measurement, Bortec Biomedical Ltd., Calgary, Canada, 1-16.

[39] Delgrosso, I., Boillat, M.-A. (1991). Carpal tunnel syndrome: Role of occupation, International Archives of Occupational and Environmental Health, Vol. 63, No. 4, 267-270, doi: 10.1007/BF00386376.

[40] Boz, C., Ozmenoglu, M., Altunayoglu, V., Velioglu, S., Alioglu, Z. (2004). Individual risk factors for carpal tunnel syndrome: An evaluation of body mass index, wrist index and hand anthropometric measurements, Clinical Neurology \& Neurosurgery, Vol. 106, No. 4, 294-299, doi: 10.1016/j.clineuro.2004.01.002.

[41] Kouyoumdjian, J.A., Zanetta, D.M.T., Morita, M.P.A. (2002). Evaluation of age, body mass index, and wrist index as risk factors for carpal tunnel syndrome severity, Muscle \& Nerve, Vol. 25, No. 1, 93-97, doi: 10.1002/mus.10007.

[42] Moghtaderi, A., Izadi, S., Sharafadinzadeh, N. (2005). An evaluation of gender, body mass index, wrist circumference and wrist ratio as independent risk factors for carpal tunnel syndrome, Acta Neurologica Scandinavica, Vol. 112, No. 6, 375-379, doi: 10.1111/j.1600-0404.2005.00528.x.

[43] Kouyoumdjian, J.A., Morita, M.P.A., Rocha, P.R.F., Miranda, R.C., Gouveia, G.M. (2000), Body mass index and carpal tunnel syndrome, Arquivos de Neuro-Psiquiatria, Vol. 58, No. 2A, 252-256, doi: 10.1590/S0004-282X200000 0200008.

[44] Douglas, C.M. (2005). Design and analysis of experiments, (6 ${ }^{\text {th }}$ edition), J. Wiley \& Soons, New York, USA. 


\title{
Analiza razširjenosti sindroma karpalnega kanala pri delavcih v proizvodnji
}

\author{
Kumar, S. , Muralidhar, M. ${ }^{\mathrm{a},{ }^{*}}$ \\ ${ }^{a}$ Department of Mechanical Engineering, North Eastern Regional Institute of Science and Technology, Itanagar, Arunachal \\ Pradesh, India
}

\section{POVZETEK}

Sindrom karpalnega kanala (angl. CTS) je najpogostejša z delom povezana mišičnoskeletna motnja zgornjih okončin. V prispevku je prikazana primerjava pogostosti CST in povezanih faktorjev tveganja med običajnimi proizvodnimi delavci in proizvodnimi delavci na montažni liniji, ki so imeli določeno raven ergonomsko urejenega delovnega mesta. Industrijska delovna okolja so bila proučena s pomočjo anketnih vprašalnikov in fizičnimi testi. Za statistično analizo podatkov sta bila uporabljena Fischerjev natančni test in površinska elektromiografija (angl. sEMG). Rezultati so bili naslednji: odrevenelost se je pojavila pri $80 \%$ običajnih delavcev in $16.66 \%$ delavcev z ergonomskimi prilagoditvami delovnega mesta, ščemenje je bilo pri prvih navzoče pri $50 \%$, pri drugih pa pri $8.33 \%$, težave pri prijemanju pa pri $80 \%$ iz prve skupine in $20 \%$ iz druge. Tinelov in Phalenov znak je pokazal podoben trend. Rezultati so pokazali, da imajo običajni proizvodni delavci več CTS simptomov kot pa delavci z nekaterimi ergonomskimi prilagoditvami delovnega mesta. Analiza sEMG signalov je razkrila, da manjša mišična aktivnost (EMG-RMS vrednosti) prispeva k CTS simptomom pri običajnih delavcih v proizvodnji. Ugotovljena je bila signifikantna razlika v EMG-RMS vrednostih CTS simptomov in kontrolnih posameznikov v prvi in drugi skupini delavcev. Ugotovljeno je bilo, da je pri posameznikih, ki imajo CS simptome vrednost signala $0.01223 \mathrm{mV}, \mathrm{v}$ primeru druge skupine pa $0.02625 \mathrm{mV}$, za kontrolne posameznike pa je bila vrednost signala $0.15614 \mathrm{mV}$ in $0.17563 \mathrm{mVs}$.

\section{PODATKI O ČLANKU}

Ključne besede:

Delavci v proizvodnji

Mišičnoskeletne motnje

Sindrom karpalnega kanala

Fisherjev eksaktni test

Površinska elektromiografija

*Kontaktna oseba:

mm@nerist.ac.in

(Muralidhar, M.)

Zgodovina članka:

Prejet 3. avgusta 2015

Popravljen 24. januarja 2016

Sprejet 8. marca 2016 